\title{
High Permittivity CPW-SIW Power Divider for Antenna Feed Networks in Airborne Phased Arrays Applications
}

\author{
Diego Lorente ${ }^{1}$, Alicja Schreiber ${ }^{1}$, Markus Limbach ${ }^{1}$, Héctor Esteban ${ }^{2}$, Vicente E. Boria ${ }^{2}$ \\ ${ }^{1}$ SAR-Technology, Microwaves and Radar Institute, German Aerospace Center, Weßling, Germany, diego.lorentecatalan@dlr.de \\ ${ }^{2}$ Departamento de Comunicaciones, Universitat Politècnica de València, Valencia, Spain
}

\begin{abstract}
An extremely compact design of a power divider in Substrate Integrated Waveguide (SIW) and fed by means of a Coplanar Waveguide (CPW) line is presented in this paper. The proposed solution presents a relative low insertion loss and good adaptation (measurements show an S11 better than $-20 \mathrm{~dB}$ for the centre frequency and below $-15 \mathrm{~dB}$ for a $30 \%$ bandwidth) in X-band, despite the use of a substrate with a high dielectric permittivity $\left(\varepsilon_{r}=9.8\right)$. In order to validate the design, various topologies of the power divider are manufactured, measured and compared with the simulated data. These power splitters will be implemented in the feed network of a phased array for an airborne radar system with Digital Beamforming capabilities operated by the German Aerospace Center.
\end{abstract}

Keywords - power divider, SIW, CPW, phased array feed networks, airborne radar.

\section{INTRODUCTION}

There has been a continuous and increasing demand for phased array systems, and their rising applications will reinforce this trend. One of these promising uses is in the field of remote-sensing, where Synthetic Aperture Radar (SAR) has become an important application. Airborne SAR sensors provide a key scope of research in order to test and experiment new systems and to support future space missions [1].

The design of the feeding network is essential for the implementation of the phased array systems, where the technology and also the topology play a key role in order to exploit the restricted available space on the aircraft in the most efficient way. Not only a good electrical performance is desired, but also a high density of integration has to be achieved in order to fulfill the system requirements.

Thanks to its conformability, integration and low cost, feed networks for airborne phased array systems are typically designed in planar technologies such as microstrip ([2],[3]) or stripline [4]. For dual polarized antennas, some special feeding configurations and interconnections may have to be considered [5].

As an alternative to microstrip, Coplanar Waveguide $(\mathrm{CPW})$ presents less attenuation since the fields do not entirely propagate through the substrate, being less sensitive to its related losses. In addition, CPW also has lower radiation losses.

Another solution to implement the antenna feed network is based on Substrate Integrated Waveguide (SIW). This technology presents the advantages of classic waveguides (high quality factor, good shielding, power handling and low cost), and at the same time it provides a high density of integration as other planar circuits, allowing us to reduce the size of the structure by using substrates with high dielectric permittivity. These properties make SIW suitable to be used for feeding network applications ([6],[7])

In this paper, a novel feeding scheme by using high permittivity power dividers in SIW technology and excited by a CPW line is presented. This design is implemented in the antenna feeding subsystem of a phased array airborne SAR sensor, and it performs a key aspect of the front-end by allowing a flexible feeding of different antenna arrangements. Due to the restricted available space in the aircraft for the antenna system, a solution with a high degree of compactness is required, thus having chosen the SIW technology for their implementation.

Each power divider is fed by means of a CPW line, which leads to the use of a broadband transition between CPW and SIW. The CPW feeding reduces the system losses, and also allows to excite the structure with a surface mounted SMP connector, which is essential in order to maintain compact the whole antenna feeding system.

Despite the use of a substrate with a high dielectric permittivity value $\left(\varepsilon_{r}=9.8\right)$, and its implementation in two different technologies, these power dividers present a very good performance in X-band. The measurements show a low insertion losses and a good reflection coefficient at the design centre frequency, as well as a bandwidth of $30 \%$ with a return loss level below $-15 \mathrm{~dB}$ in an extremely compact size.

This work is structured as follows: first, the various topological solutions proposed for feeding the antenna system with CPW-SIW power dividers are discussed. Then, the design of a SIW power divider, and its excitation by means of a CPW line, will be explained. Consequently, the design will be validated by comparing the full-wave simulated data with measurements of some manufactured prototypes. Finally, the conclusions and future work will be discussed.

\section{Antenna FeEd System}

For a phased array system, a beam steering of the antenna radiation pattern, both in azimuth and elevation, is desired. 


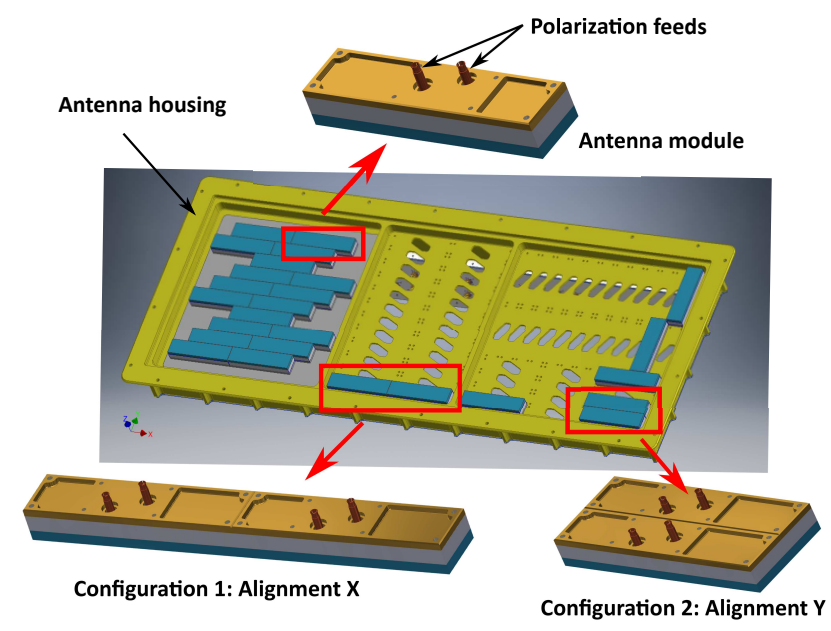

Fig. 1. Antenna housing and configuration examples.

This is achieved by means of different antenna modules that are mechanically rearranged in horizontal or vertical direction, as it is depicted in Figure 1. Each antenna module is composed of 4 dual polarized microstrip patches in X-band, operating at $9.6 \mathrm{GHz}$ and with a bandwidth of $1 \mathrm{GHz}$. These antenna configurations lead to different feeding schemes, that are implemented using three geometrical variations of the power divider, as it can be seen in Figure 2.

Due to the common restriction of the available space in the aircraft, a compact solution for the implementation of the feeding system has to be considered. In addition, the design of the power dividers in SIW allows their miniaturization by using a high dielectric substrate. Since it is also an electrically closed structure, it is possible to stack vertically two of them to feed the two polarizations (horizontal and vertical) of two antenna modules in the same space, thus taking advantage more efficiently of the available area.

\section{CPW-SIW POWER DIVIDERS}

In order to feed all the possible configurations needed for different antenna modules arrangements, three power dividers have to be considered. These three power splitters are geometrical variations of a 2 -way balanced T-junction divider with an inductive post, designed in SIW and fed by a CPW line. These three power dividers will be named as $\mathrm{H}, \mathrm{V}$ and $\mathrm{HV}$ depending on a given antenna alignment if they are used to feed only one polarization (H or $\mathrm{V}$ ) or both (HV).

The input and output connectors of the power dividers lay in different sides of the structure, since it enables to have the divider input on top and the outputs feeding the antenna modules on bottom. Proceeding in this way, a stacked assembly can be obtained.

\section{A. SIW Design}

The SIW design is implemented using a Rogers TMM10i substrate with a high dielectric permittivity $\left(\varepsilon_{r}=9.8\right)$, dielectric loss tangent of 0.002 and thickness of $1.905 \mathrm{~mm}$. Since the power dividers have to feed the antenna modules, they are designed to work at a center frequency of $9.6 \mathrm{GHz}$

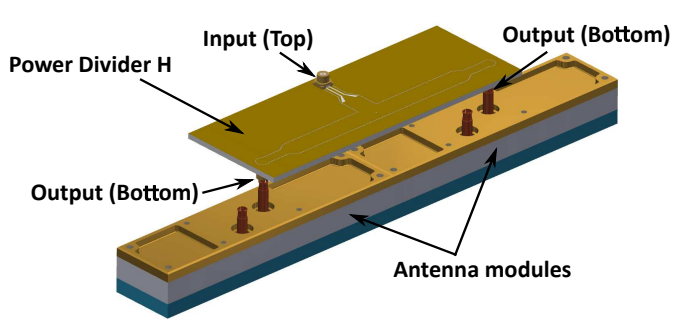

(a)

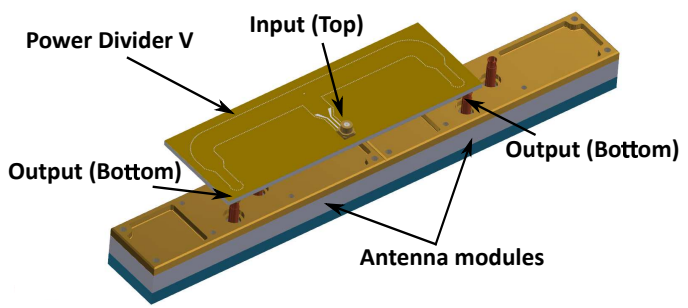

(b)

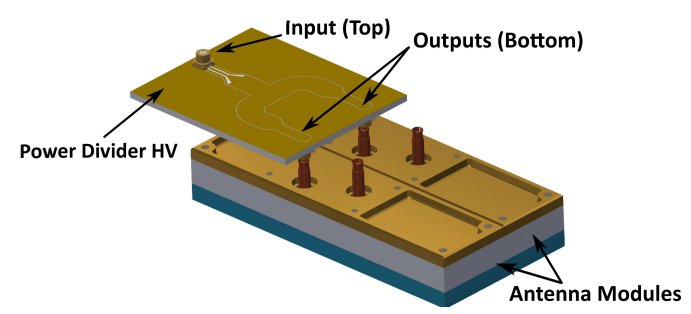

(c)

Fig. 2. (a) Configuration 1 fed by Power Divider H; (b) Configuration 1 fed by Power Divider V; (c) Configuration 2 fed by Power Divider HV

Table 1. SIW values

\begin{tabular}{|c||c||c|}
\hline Substrate dielectric permittivity & $\varepsilon_{r}$ & 9.8 \\
\hline Substrate thickness & $h$ & $1.905 \mathrm{~mm}$ \\
\hline SIW line width & $a_{\text {siw }}$ & $8 \mathrm{~mm}$ \\
\hline Distance between vias & $p_{\text {vias }}$ & $0.65 \mathrm{~mm}$ \\
\hline Vias diameter & $d_{\text {via }}$ & $0.4 \mathrm{~mm}$ \\
\hline Center via diameter & $d_{\text {via } 2}$ & $0.5 \mathrm{~mm}$ \\
\hline Center via offset & $d_{\text {offset }}$ & $3.58 \mathrm{~mm}$ \\
\hline
\end{tabular}

with a bandwidth of $1 \mathrm{GHz}$. As a balanced divider, the same amplitude (power) and phase levels must be achieved for each output. The inductive post at the center of the proposed topology compensates the capacitance added by the T-junction. The geometrical values of the SIW power divider are summarized in Table 1 and displayed in Figure 3.

\section{B. CPW Feeding}

The proposed SIW structure is fed by means of a $50 \Omega$ grounded CPW line. This CPW feeding allows exciting the structure with a surface mounted SMP connector [8], which is a novel type of SMA connector. This type of connectors present a small size and it can be slightly bended, since they have a radial tolerance that makes them suitable for high vibration applications, as it usually occurs during a flight. They provide an easy interconnection to the antenna modules just by 


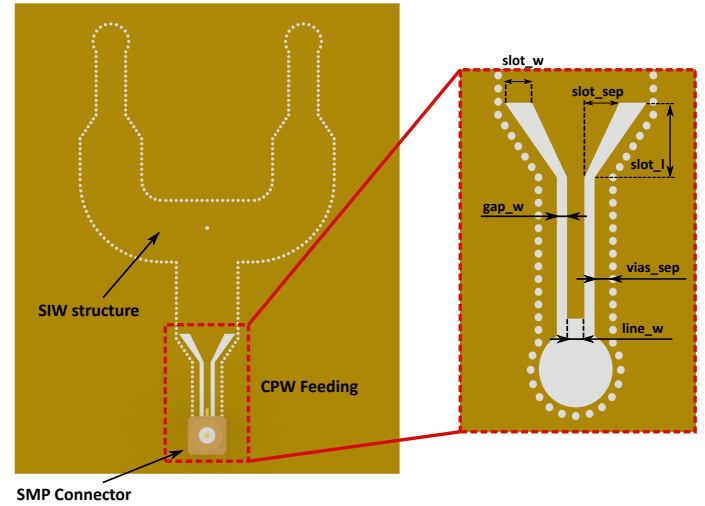

(a)

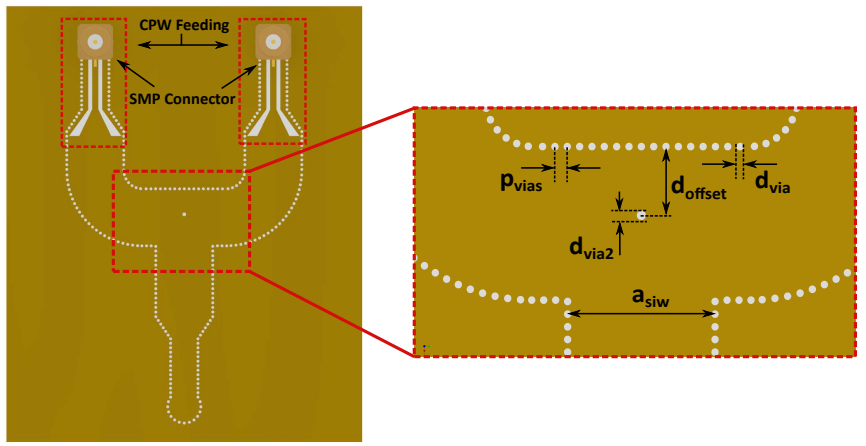

(b)

Fig. 3. Power divider HV (a) Top view: CPW feeding parameters; (b) Bottom view: SIW parameters.

pressing them. Thus, the use of intermediate cables is avoided and a more compact system can be achieved.

Due to the CPW feeding line, a transition to SIW is required. Some previous works use a tapered microstrip transition to excite the SIW structure ([9],[10]). In this work, a broadband transition between CPW and SIW is used [11]. In comparison with other types of excitations such as microstrip, the feeding of the structure by means of a CPW line improves the insertion losses, since the CPW electromagnetic fields do not propagate entirely through the substrate, thereby reducing the impact of the substrate losses. Besides, radiation losses are lower in CPW technology.

The feeding line is designed to have a $50 \Omega$ impedance, and it is fed by a connector SMP Model 19S103-500L5 from Rosenberger [8]. The design values of the CPW line and the transition are listed in Table 2 and shown in Figure 3.

The power divider design steps were performed as follows: first the dielectric filled T-junction waveguide divider with inductive post was designed. Consequently, the equivalent SIW model of such structure was implemented. The feeding CPW line and the transition CPW-SIW were separately designed. Finally, the completed SIW structure with the CPW feeding line was simulated and properly optimized.

The analysis and optimization process was performed by HFSS v.2019 R3, a software package of the Ansys Electromagnetics Desktop.
Table 2. CPW feeding line values

\begin{tabular}{|c||c||c|}
\hline CPW line width & line_w & $0.9 \mathrm{~mm}$ \\
\hline Gap width & gap_w & $0.52 \mathrm{~mm}$ \\
\hline Vias separation & vias_sep & $0.95 \mathrm{~mm}$ \\
\hline Slot length & slot_l & $3.7 \mathrm{~mm}$ \\
\hline Slot width & slot_w & $1.4 \mathrm{~mm}$ \\
\hline Slot separation & slot_sep & $1.8 \mathrm{~mm}$ \\
\hline
\end{tabular}

\section{Manufactured prototypes}

The manufactured power dividers can be seen in Figure 4. A comparison between simulation and measurement results for each design is shown in Figure 5. The measurements show a good agreement with the simulated data. The measured reflection coefficient $\mathrm{S} 11$ of each power divider is below $-15 \mathrm{~dB}$, not only for the desired frequency band (9.1-10.1 GHz) but beyond, with a bandwidth of up to $30 \%$ below this value. The measured matching level at the design center frequency $(9.6 \mathrm{GHz})$ for the three prototypes is better than $-20 \mathrm{~dB}$.

The insertion loss level varies depending on the electrical length of each power divider. The simulation show values between 3.5-3.8 $\mathrm{dB}$. These values consider the substrate and conductor losses, the insertion losses due to the connectors and the transition. The measurements also includes the effect of the SMA-SMP adapters which are required for the measurement. This explains the 0.3-0.4 dB difference between simulation and measurement.

The measured insertion losses also show a light standing wave due to a missmatch at the output ports. This effect is especially visible in the case of the power divider V. It can be explained because of unaccuracies at the connectors soldier or due to fabrication tolerances.

A comparison between the proposed CPW-SIW power divider and some other similar designs with lower dielectric permitivities is summarized in Table 3. The measured prototypes present at least as good matching level as the other designs, and a relative low insertion loss level despite using a high dielectric permittivity, which leads to an extremely compact implementation. The proposed work is the only one fed by SMP connectors.

Table 3. Comparative analysis of power dividers

\begin{tabular}{|c||c||c||c||c||c|}
\hline Ref & Frequency & Feeding & $\varepsilon_{r}$ & S11 & S21\&S31 \\
\hline$[12]$ & $5-11 \mathrm{GHz}$ & $\mathrm{CPW}$ & 3.38 & $-11 \mathrm{~dB}$ & $-3.5 \mathrm{~dB}$ \\
\hline$[13]$ & $58-63 \mathrm{GHz}$ & $\mathrm{CPW}$ & 2.2 & $-15 \mathrm{~dB}$ & $-3.1 \mathrm{~dB}$ \\
\hline$[10]$ & $2-3.5 \mathrm{GHz}$ & Microstrip & 6.15 & $-14 \mathrm{~dB}$ & $-4.1 \mathrm{~dB}$ \\
\hline$[14]$ & $11-15 \mathrm{GHz}$ & Microstrip & 2.2 & $-15 \mathrm{~dB}$ & $-3.5 \mathrm{~dB}$ \\
\hline$[15]$ & $8-12.5 \mathrm{GHz}$ & Microstrip & 2.2 & $-15 \mathrm{~dB}$ & $-3.4 \mathrm{~dB}$ \\
\hline This work & $\mathbf{8 - 1 1} \mathbf{G H z}$ & $\mathbf{C P W}$ & $\mathbf{9 . 8}$ & $\mathbf{- 1 5} \mathbf{d B}$ & $\mathbf{- 3 . 6} \mathbf{~ d B}$ \\
\hline
\end{tabular}

\section{CONCLUSION}

A really compact SIW power divider fed by a CPW line was presented. The design was validated by means of measurements of three different manufactured prototypes. 


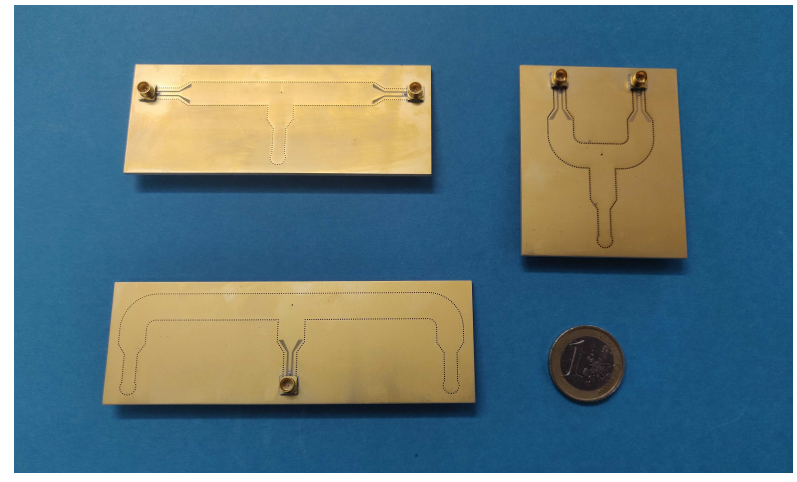

Fig. 4. Manufactured prototypes.

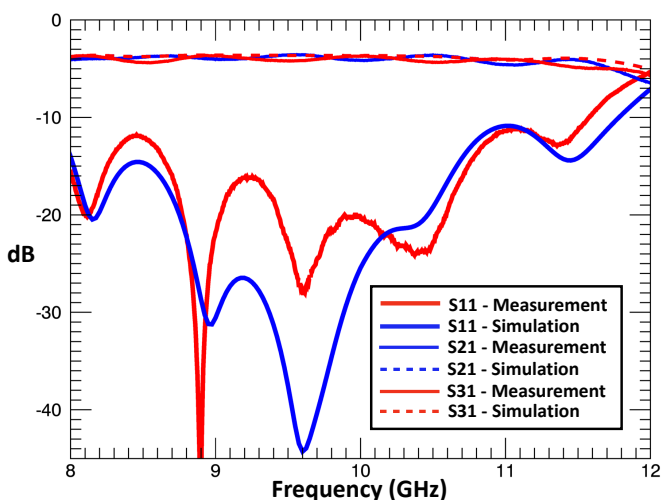

(a)

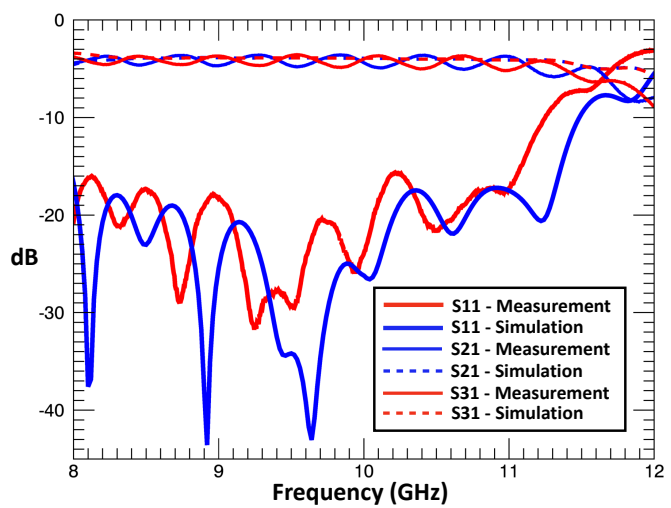

(b)

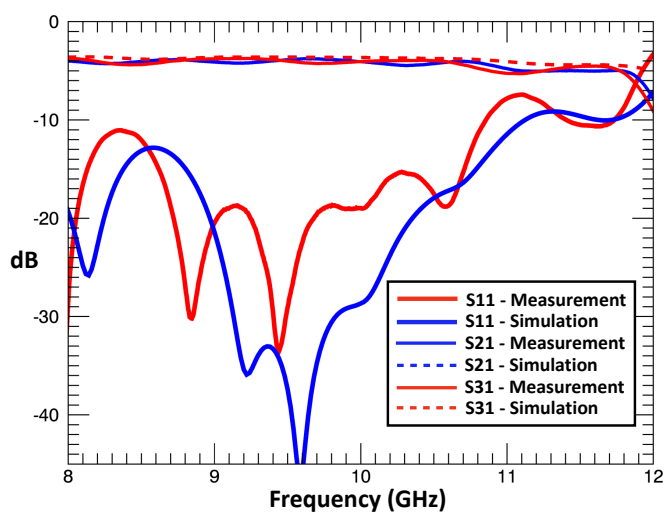

(c)

Fig. 5. Comparison simulation and measurement results (a) Power Divider H; (b) Power Divider V; (c) Power Divider HV
Despite the use of a high dielectric permittivity and the implementation using two technologies (CPW and SIW), this solution presents a really good electrical performance, which makes it suitable to feed extremely compact phased array systems. In addition, due to the use of a CPW feeding, the structure can be excited by means of a surface mounted connector, thus removing the possibility of using a pin connector inside the substrate to excite the SIW structure, and this way, making the design more robust against connector feeding tolerances. These power dividers will be used in the airborne digital beamforming SAR system (DBFSAR) of the German Aerospace Center.

\section{REFERENCES}

[1] A. Reigber et al., "DBFSAR: An airborne very high-resolution digital beamforming SAR system," 14th European Radar Conference, Nuremberg, Germany, Oct. 2017.

[2] A. S. Ali, M. Raza, and M. A. Shah, "Design \& development of a 32 elements X-band phased array antenna for airborne \& space-borne SAR payloads," 2012 9th International Bhurban Conference on Applied Sciences \& Technology (IBCAST), Islamabad, Pakistan, Jan. 2012.

[3] A. Moiz et al., "Design \& development of linearly polarized C-band phased array antenna for airborne \& space-borne SAR payloads," 2014 11th International Bhurban Conference on Applied Sciences \& Technology (IBCAST), Islamabad, Pakistan, Jan. 2012.

[4] M. Limbach et al., "S-Band antenna for airborne polarimetric and interferometric SAR-applications," 7th European Conference on Antennas and Propagation, Gothenburg, Sweden, April 2013.

[5] J. Choi et al., "Composite right/left-Handed (CRLH) phased-array feed network for frequency scanning antenna," 42nd European Microwave Conference, Amsterdam, The Netherlands, Oct. 2012.

[6] T. Djerafi and K. Wu, "Substrate integrated waveguide feeding network for angular-limited scan arrays with overlapped subarrays," IEEE MTT-S International Microwave Workshop Series on Advanced Materials and Processes, Pavia, Italy, Sept. 2017.

[7] Y. Ji et al., "Reconfigurable phased-array antenna using continuously tunable substrate integrated waveguide phase shifter," IEEE Trans. Antennas Propag., vol. 67, no. 11, Nov. 2019.

[8] SMP, longwipe-SMP, mini-SMP connectors. [Online]. Available: https://www.rosenberger.com/0_documents/de/catalogs/ba_communicati on/catalog_coax/02_Chapter_SMP_Longwipe-SMP_Mini-SMP.pdf

[9] S. Datta et al., "Design of broadband power divider based on substrate-integrated waveguide technology," IEEE Applied Electromagnetics Conference, Bhubaneswar, India, Dec. 2013.

[10] E. Setijadi and A. Pandu, "Ultrawide band power divider based on substrate integrated waveguide (SIW) for S-band applications," ARPN Journal of Engineering and Applied Sciences, vol. 13, no. 1, Jan. 2018.

[11] R. Kazemi, A. E. Fathy, S. Yang, and R. A. Sadeghzadeh, "Development of an altra wide band GCPW to SIW transition," IEEE Radio and Wireless Symposium, Santa Clara, USA, Jan. 2012.

[12] R. Kazemi and A. E. Fathy, "Design of single-ridge SIW power dividers with over 75\% bandwidth," IEEE MTT-S International Microwave Symposium, Tampa, USA, June 2014.

[13] K. B. Kumar and T.Shanmuganantham, "3-port T-junction SIW power divider for $60 \mathrm{GHz}$ applications," IEEE International Conference on Antenna Innovations \& Modern Technologies for Ground, Aircraft and Satellite Applications (iAIM), Bangalore, India, Nov. 2017.

[14] M. Ramesh and S. Raghavan, "Design and implementation of substrate integrated waveguide power divider for X- and Ku-band application," South Asian Journal of Research in Engineering Science and Technology, vol. 3, no. 11, Nov. 2018.

[15] A. R. Akbarzadeh and Z. Shen, "Waveguide power dividers using multiple posts," Microw. Opt. Techn. Let., vol. 50, no. 4, April 2008. 